\title{
Hard and soft probe - medium interactions in a 3D hydro+micro approach at RHIC
}

\author{
S.A. Bass ${ }^{1}$, T. Renk ${ }^{2,3}$, J. Ruppert ${ }^{4}$ and C. Nonaka ${ }^{5}$ \\ ${ }^{1}$ Department of Physics, Duke University, Durham, North Carolina 27708-0305, USA \\ ${ }^{2}$ Department of Physics, PO Box 35 FIN-40014 University of Jyväskylä, Finland \\ ${ }^{3}$ Helsinki Institute of Physics, PO Box 64 FIN-00014 University of Helsinki, Finland \\ ${ }^{4}$ Department of Physics, McGill University, H3A 2T8, Montreal, Quebec, Canada \\ ${ }^{5}$ Department of Physics, Nagoya University, Nagoya 464-8602, Japan \\ E-mail: bass@phy.duke.edu
}

Submitted to: J. Phys. G: Nucl. Phys.

PACS numbers: 25.75.-q, 12.38.Mh

\begin{abstract}
We utilize a 3D hybrid hydro+micro model for a comprehensive and consistent description of soft and hard particle production in ultra-relativistic heavyion collisions at RHIC. In the soft sector we focus on the dynamics of (multi-)strange baryons, where a clear strangeness dependence of their collision rates and freeze-out is observed. In the hard sector we study the radiative energy loss of hard partons in a soft medium in the multiple soft scattering approximation. While the nuclear suppression factor $R_{A A}$ does not reflect the high quality of the medium description (except in a reduced systematic uncertainty in extracting the quenching power of the medium), the hydrodynamical model also allows to study different centralities and in particular the angular variation of $R_{A A}$ with respect to the reaction plane, allowing for a controlled variation of the in-medium path-length.
\end{abstract}

Experiments at the Relativistic Heavy Ion Collider (RHIC) have established a significant suppression of high- $p_{T}$ hadrons produced in central A+A collisions compared to those produced in peripheral $\mathrm{A}+\mathrm{A}$ or binary scaled $\mathrm{p}+\mathrm{p}$ reactions, indicating a strong nuclear medium effect [1, 2], commonly referred to as jet-quenching. Within the framework of perturbative QCD, the leading process of energy loss of a fast parton is gluon radiation induced by multiple soft collisions of the leading parton or the radiated gluon with color charges in the quasi-thermal medium [3, 4, 5].

Over the past two years, a large amount of jet-quenching related experimental data has become available including but not limited to the nuclear modification factor $R_{A A}$, the elliptic flow $v_{2}$ at high $p_{T}$ (as a measure of the azimuthal anisotropy of the jet cross section) and a whole array of high $p_{T}$ hadron-hadron correlations. Computations of such jet modifications have acquired a certain level of sophistication regarding the incorporation of the partonic processes involved. However, most of these calculations 
have been utilizing over-simplified models for the underlying soft medium, e.g. assuming a simple density distribution and its variation with time. Even in more elaborate setups, most jet quenching calculations assume merely a one- or two-dimensional Bjorken expansion.

The availability of a three-dimensional hydrodynamic evolution code [6] and related hybrid approaches allow for a much more detailed study of jet interactions in a longitudinally and transversely expanding medium. The variation of the gluon density in these approaches is very different from that in a simple Bjorken expansion. A previous calculation in this direction [7, 8] estimated the effects of 3-D expansion on the $R_{A A}$. However, this approach treated the energy loss of jets in a rather simplified and heuristic manner. Here, we shall perform a detailed investigation of the modification of jets in a three dimensionally expanding medium within the BDMPS formalism utilizing quenching weights as described in [9]. In addition, 3-D hydrodynamic and hybrid models have been very successful in describing the majority of features of soft particle production at RHIC (with HBT interferometry being the sole exception) - this we shall utilize in order to determine the medium properties for the jet-quenching calculation.

In this write-up, we utilize a state-of-the-art fully 3-D hybrid hydro+micro transport model [6]. The model employs relativistic 3D-hydrodynamics for the early, dense, deconfined stage of the reaction and a microscopic non-equilibrium model for the later hadronic stage where the equilibrium assumptions are not valid anymore. It is capable of self-consistently calculating the freezeout of the hadronic system, while accounting for the collective flow on the hadronization hypersurface generated by the QGP expansion. The initial conditions of the hydrodynamic calculation are tuned to describe the hadronic data in the soft sector, such as hadron yields, spectra, rapiditydistributions as well as radial and elliptic flow. Note that while the modeling of the hadronic stage is of paramount importance for the proper description of the medium in the soft sector, it does not contribute to the jet energy loss.

In the left frame of Fig. 1 we analyze the $P_{T}$ spectra of multistrange baryons. Our results show good agreement with experimental data for $\Lambda, \Xi, \Omega$ from the STAR collaboration [10, 11]. Recent experimental results suggest that at thermal freezeout multistrange baryons exhibit less transverse flow and a higher temperature closer to the chemical freezeout temperature compared to non- or single-strange baryons [10, 11]. This behavior can be understood in terms of the flavor dependence of the hadronic cross section, which decreases with increasing strangeness content of the hadron. The reduced cross section of multi-strange baryons leads to a decoupling from the hadronic medium at an earlier stage of the reaction, allowing them to provide information on the properties of the hadronizing QGP less distorted by hadronic final state interactions [12, 13, 14]. In microscopic calculations the early decoupling will manifest itself via a reduced number of collisions for the respective hadron species. It should be noted that the analogous behavior has already been observed in experiments at the CERN-SPS [15, 16, 17, 18, 19].

The pseudo-rapidity dependence of the number of hadronic rescatterings for 

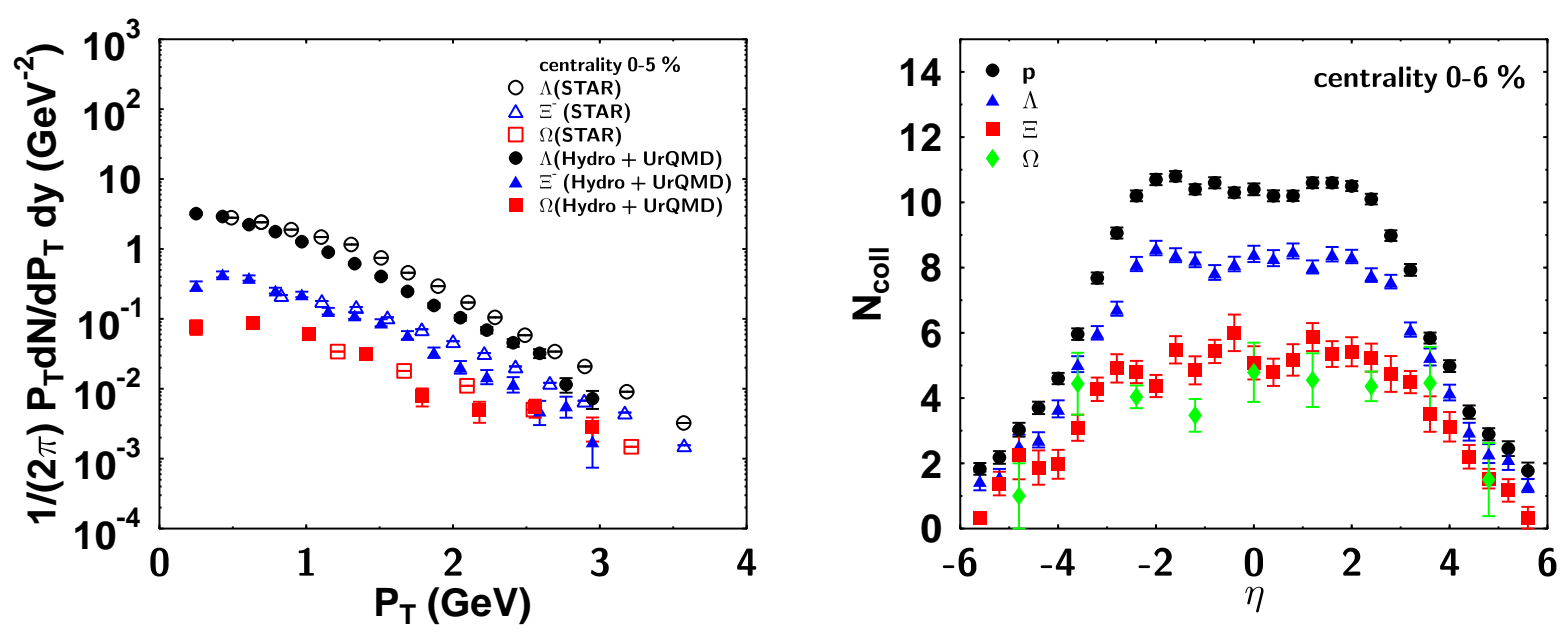

Figure 1. Left: $P_{T}$ spectra of (multi-)strange baryons calculated in a hybrid hydro+micro approach compared to STAR data. Right: pseudo-rapidity distribution of the number of rescatterings for baryons of varying strange quark content.

different baryon species can be used to corroborate the above findings and is analyzed in the right frame of Fig. 1, which shows the number of collisions of $p, \Lambda, \Xi$ and $\Omega$ as a function of $\eta$ at $b=2.4,4.5$ and $6.3 \mathrm{fm}$. The distributions appear to be similar to that of the particle yield psuedorapidity distribution. At midrapidity we find a plateau region extending from $\eta=-3$ to 3 , followed by a steep drop-off to forward and backward rapidities. The flavor dependence of the average collision numbers is again clearly seen, even though we would like to point out that the shapes of the different distributions are very similar. The large plateau region indicates the rapidity domain in which interacting matter can be found and in which the application of thermodynamic concepts is viable.

Having determined the properties and dynamics of the soft sector, we can now utilize the time-evolution of the medium provided by our model for the calculation of jet energy-loss. Thus, our calculation significantly reduces the systematic uncertainties usually associated with the medium parametrization and allows for a precision calculation of all effects associated with hard probe - medium interactions. Our calculation follows the BDMPS formalism for radiative energy loss [20] using quenching weights as introduced by Salgado and Wiedemann [21, 9].

In [22, 23] it has been shown that $R_{A A}$ for central collisions only constrains a scale, but not the detailed functional form of energy loss probability distribution $\langle P(\Delta E)\rangle_{T_{A A}}$. In the approach outlined above, this is manifest in the parameter $K$ in the expression for the local transport coefficient $\hat{q}(\xi)$ :

$$
\hat{q}(\xi)=K \cdot 2 \cdot \epsilon^{3 / 4}(\xi)
$$

which then was adjusted to the data in central collisions. We illustrate in the left frame of Fig. 2 that three different dynamical models, a 2D hydrodynamical evolution [24], the 3D hydrodynamical evolution outlined above [6] and a parametrized fireball 

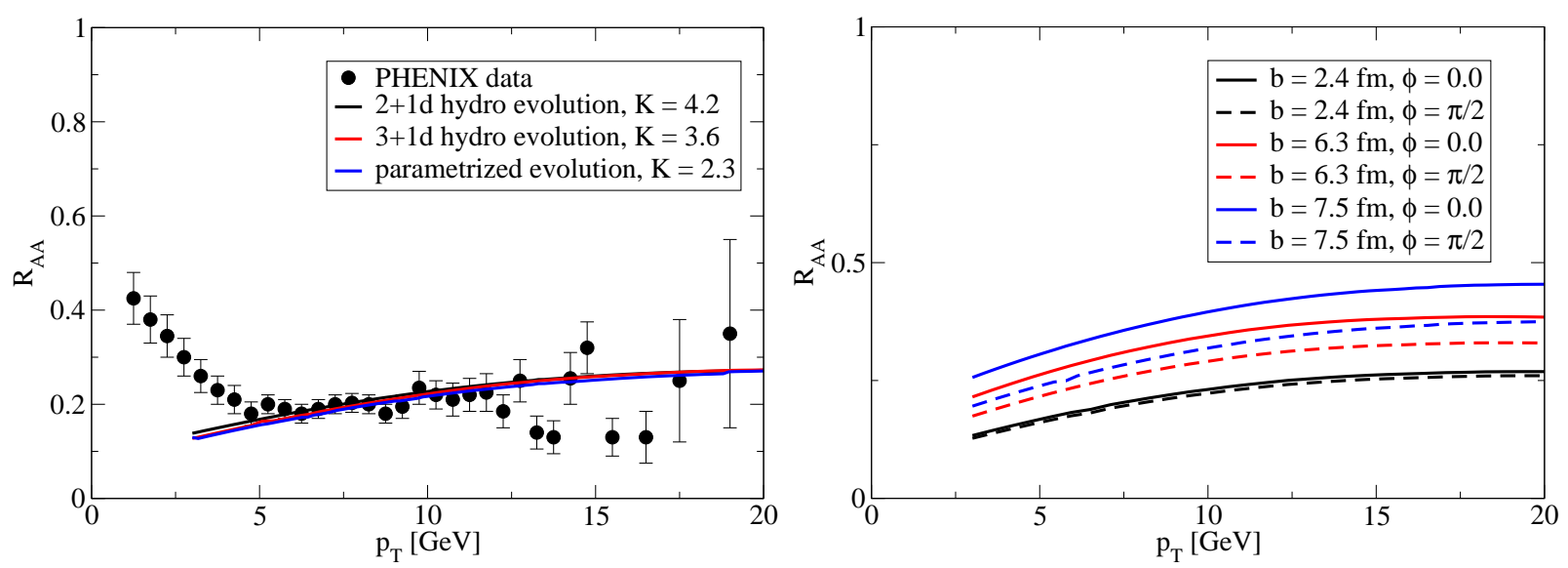

Figure 2. Left: $R_{A A}$ for central collisions as calculated in three different models for the medium evolution with the overall quenching power scale $K$ adjusted to data. Right: $p_{T}$ dependence of $R_{A A}$ in plane (solid) and out of plane (dashed) emission at different values of impact parameter $\mathbf{b}$.

evolution [25] give almost equal descriptions of $R_{A A}$ once the scale parameter is adjusted, albeit they require different values of $K$ (the chief reason for this being the different longitudinal dynamics). The $\pm 50 \%$ spread in the values of $K$ for the different models of the medium is a measure for the systematic error inherent in the tomographic analysis of jet energy-loss via the nuclear modification function $R_{A A}$.

However, one may gain predictive power in going to collisions at finite impact parameter b. In particular, the $\phi$ dependence of $R_{A A}$ for non-central collisions constitutes a systematic variation of path-length within a system with fixed overall scale. The average path-length is expected to be smaller for a parton emitted in plane as compared to one emitted out of plane, and hence $R_{A A}$ is expected to be larger at $\phi=0$ than at $\phi=\pi / 2$ with the difference in $R_{A A}$ between these angles increasing with the initial asymmetry (and hence $\mathbf{b}$ ). Using a simple model for the time-evolution of the medium and collective flow effects, it has been shown in [26] that the $\phi$ dependence of $R_{A A}$ is quite sensitive to the initial gluon density distribution and temporal evolution of the medium.

Utilizing the previously discussed 3-D RFD model [6], we show the $p_{T}$ dependence of $R_{A A}$ for emission in plane and out of plane at three different impact parameters $\mathbf{b}$ in the right frame of Fig. 2. As expected, $R_{A A}$ grows for more peripheral collisions as there is less soft matter produced to induce energy loss. Moreover, there is a smooth angular variation of $R_{A A}$ observed, reflecting the underlying medium asymmetry. The difference between in-plane and out of plane emission grows with impact parameter, at $\mathbf{b}=2.4 \mathrm{fm}$ there is hardly angular variation whereas at $7.5 \mathrm{fm}$ differences are of order $20 \%$.

In summary, we have utilized a 3D hybrid hydro+micro model for a comprehensive and consistent description of soft and hard particle production in ultra-relativistic heavyion collisions at RHIC. In the soft sector we have focused on the dynamics of (multi- 
Hard and soft probe - medium interactions in a $3 D$ hydro+micro approach at RHIC

)strange baryons, where a clear strangeness dependence of their collision rates and freeze-out is observed. In the hard sector we have studied the radiative energy loss of hard partons in a soft medium in the multiple soft scattering approximation. Our analysis should be seen as the starting point for a comprehensive study of probe-medium interactions treating the hard and soft sector on equal footing.

\section{Acknowledgments}

This work was supported in part by an Outstanding Junior Investigator Award from the U. S. Department of Energy (grant DE-FG02-03ER41239). TR was supported in

part by the Academy of Finland, Project 206024 and JR acknowledges support by the Natural Sciences and Engineering Research Council of Canada.

\section{References}

[1] Adcox K et al. (PHENIX) 2002 Phys. Rev. Lett. 88022301 (Preprint nucl-ex/0109003)

[2] Adler C et al. (STAR) 2002 Phys. Rev. Lett. 89202301 (Preprint nucl-ex/0206011)

[3] Gyulassy M and Wang X n 1994 Nucl. Phys. B420 583-614 (Preprint nucl-th/9306003)

[4] Baier R, Dokshitzer Y L, Mueller A H, Peigne S and Schiff D 1997 Nucl. Phys. B483 291-320 (Preprint hep-ph/9607355)

[5] Zakharov B G 1997 JETP Lett. 65 615-620 (Preprint hep-ph/9704255)

[6] Nonaka C and Bass S A 2007 Phys. Rev. C75 014902 (Preprint nucl-th/0607018)

[7] Hirano T and Nara Y 2002 Phys. Rev. C66 041901 (Preprint hep-ph/0208029)

[8] Hirano T and Nara Y 2004 Phys. Rev. C69 034908 (Preprint nucl-th/0307015)

[9] Salgado C A and Wiedemann U A 2003 Phys. Rev. D68 014008 (Preprint hep-ph/0302184)

[10] Estienne M (STAR) 2005 J. Phys. G31 S873-S880 (Preprint nucl-ex/0412041)

[11] Adams J et al. (STAR) 2004 Phys. Rev. Lett. 92182301 (Preprint nucl-ex/0307024)

[12] van Hecke H, Sorge H and Xu N 1998 Phys. Rev. Lett. 81 5764-5767 (Preprint nucl-th/9804035)

[13] Dumitru A, Bass S A, Bleicher M, Stoecker H and Greiner W 1999 Phys. Lett. B460 411-416 (Preprint nucl-th/9901046)

[14] Teaney D, Lauret J and Shuryak E V 2001 (Preprint nucl-th/0110037)

[15] Jones P G et al. (NA49) 1996 Nucl. Phys. A610 188c-199c

[16] Bormann C et al. (NA49) 1997 J. Phys. G23 1817-1825

[17] Appelshauser H et al. (NA49) 1998 Phys. Lett. B444 523-530 (Preprint nucl-ex/9810005)

[18] Andersen E et al. (WA97) 1999 J. Phys. G25 181-188

[19] Andersen E et al. 1998 Phys. Lett. B433 209-216

[20] Baier R, Dokshitzer Y L, Mueller A H, Peigne S and Schiff D 1997 Nucl. Phys. B484 265-282 (Preprint hep-ph/9608322)

[21] Salgado C A and Wiedemann U A 2002 Phys. Rev. Lett. 89092303 (Preprint hep-ph/0204221)

[22] Renk T and Eskola K J 2006 (Preprint hep-ph/0610059)

[23] Renk T 2006 Phys. Rev. C74 034906 (Preprint hep-ph/0607166)

[24] Eskola K J, Honkanen H, Niemi H, Ruuskanen P V and Rasanen S S 2005 Phys. Rev. C72 044904 (Preprint hep-ph/0506049)

[25] Renk T 2004 Phys. Rev. C70 021903 (Preprint hep-ph/0404140)

[26] Majumder A 2006 (Preprint nucl-th/0608043) 